\title{
Role of reactive nitrogen species in male infertility
}

\author{
Sejal B Doshi, Karishma Khullar, Rakesh K Sharma and Ashok Agarwal ${ }^{*}$
}

\begin{abstract}
Reactive nitrogen species (RNS) is a subset of free oxygen radicals called reactive oxygen species (ROS). Physiological levels of ROS are necessary to maintain the reproductive functions such as cell signaling, tight junction regulation, production of hormones, capacitation, acrosomal reaction, sperm motility, and zona pellucida binding. However, an excess of RNS can adversely affect reproductive potential by causing testicular dysfunction, decreased gonadotropin secretion, and abnormal semen parameters. Because such levels of RNS have been demonstrated in males with fertility problems and routine semen analysis has not been able to accurately predict IVF outcomes, it is imperative that novel strategies be developed in order to both assess and treat oxidative stress. This article describes both physiological and pathological roles of this unique subset of ROS.
\end{abstract}

Keywords: Infertility, Nitric oxide, Reactive nitrogen species, Oxidative stress

\section{Background}

Reactive Oxygen Species (ROS) comprise a class of radical and nonradical oxygen derivatives that play a significant role in reproductive biology. Because they have an unpaired electron in their outer orbit, ROS are highly reactive and interact with a variety of lipids, proteins, and nucleic acids in the body [1-12]. Such reactions are not only harmful for reproductive potential, but they also generate more free radicals, thereby perpetuating a chain of reactions and creating high amounts of oxidative stress.

Oxidative stress (OS) results from an imbalance of free radicals and antioxidant defense mechanisms in the body. Normally, antioxidants, both enzymatic and non enzymatic, scavenge free radical species and protect the body from over-exposure to oxidative stress. For instance, the antioxidant Vitamin $\mathrm{E}$ has been shown to enhance the fusion of spermatozoa with the oocyte, thereby improving zona pellucida binding. Another antioxidant that has been shown to neutralize radical-induced damage to the sperm plasma membrane is albumin. However, in certain instances, the lack of antioxidants can result in oxidative stress [11].

\footnotetext{
* Correspondence: agarwaa@ccf.org

Center for Reproductive Medicine, Cleveland Clinic, Euclid Avenue, Cleveland $95000 \mathrm{H}$, USA
}

OS has been associated with many deleterious effects on the reproductive system as well as numerous diseases, such as cancer, diabetes, varicocele, rheumatoid arthritis, AIDS, inflammation, and liver damage [4-6,10-19]. ROS, not only includes oxygen radicals such as the hydroxyl radical, superoxide radical, and hydrogen peroxide but also a subclass of nitrogencontaining compounds collectively known as reactive nitrogen species (RNS). Examples of RNS include peroxynitrite anion, nitroxyl ion, nitrosyl-containing compounds, and nitric oxide. While important for various physiological functions, RNS in excessive amounts, which contributes to nitrosative stress, may exert pathological effects on the male reproductive system [10-12,17,18,20]. In particular, RNS has recently been implicated in inducing poor sperm function and sperm fertilizing ability [21-23]. While many studies have largely focused on oxidative stress and ROS, the purpose of this paper is to address the impact of RNS on male infertility. A consolidation of the available information on RNS will allow the development of potential anti-oxidant treatments for conditions associated with nitrosative stress. Overall, through these aforementioned treatments and improvements in assisted reproductive techniques, favorable results for subfertile couples can be achieved $[15,16]$. 


\section{Sources of reactive nitrogen species}

Within the human body, a myriad of sources produce RNS, such as mesangial cells, smooth muscle cells, platelets, and hepatocytes $[12,24,25]$. However, RNS is especially prominent in different areas of the male reproductive system and these sources can be categorized by structure and various cell types such as seminal ejaculate, accessory glands, epididymis, penis, testes, and ducts [See Table 1; Figure 1].

\section{Mechanism of formation of nitric oxide}

Nitric Oxide (NO) is produced from L-arginine via Nitric Oxide Synthase (NOS). It requires oxygen and a number of cofactors such as nicotinamide adenine dinucleotide phosphate (NADPH), flavin mononucleotide (FMN), flavin adenine dinucleotide (FAD), calmodulin, and calcium, resulting in the formation of $\mathrm{NO}$ as well as a byproduct known as L-citrulline [10-12,24,25]. There are three forms of NOS which exert their effect through protein-protein interactions and catalyze the aforementioned reaction: 1 ) endothelial NOS (eNOS), 2) inducible NOS (iNOS), and 3) neuronal NOS (nNOS). Each isoform has a reductase domain that contains a compound known as tetrahydrobiopterin (BH4), which is essential for efficient production of NO [24,25]. Interestingly, a testis-specific subclass of nNOS, known as TnNOS, has been recently identified as a major contributor to the formation of NO [31-34]. TnNOS is found to localize solely in the Leydig cells of the testis, thereby suggesting its involvement in steroidogenesis. With regard to eNOS and inducible NOS, both have been shown to be structurally associated with proteins such as occludin, actin, alpha-tubulin, vimentin, suggesting their importance in controlling tight junctions in the testis. Moreover, studies have illustrated that both eNOS and iNOS are involved in germ cell apoptosis. This is shown by the fact that eNOS has been linked to degenerating germ cell lines while iNOS has been associated with maintenance of germ cell number in the seminiferous epithelium. When considering iNOS alone, studies have shown that it partly mediates alpha-fodrin proteolysis, promoting germ cell necrosis.

Usually, iNOS is expressed in most cells only after induction by immunologic and inflammatory stimuli, such as peritoneal macrophages and macrophage products, including interleukin-1 and tumor necrosis factor-alpha $[20,31,32]$. However, it has been shown that this form of NOS can be induced by factors released from round spermatids, implicating a regulatory role of germ cells on Sertoli and Leydig cell NOS function [20,34]. Overall, these three isoforms are found in various cells of the testis, including Sertoli cells, germ cells in the seminiferous epithelium,

Table 1 Location of nitric oxide/nitric oxide synthase in the male reproductive system

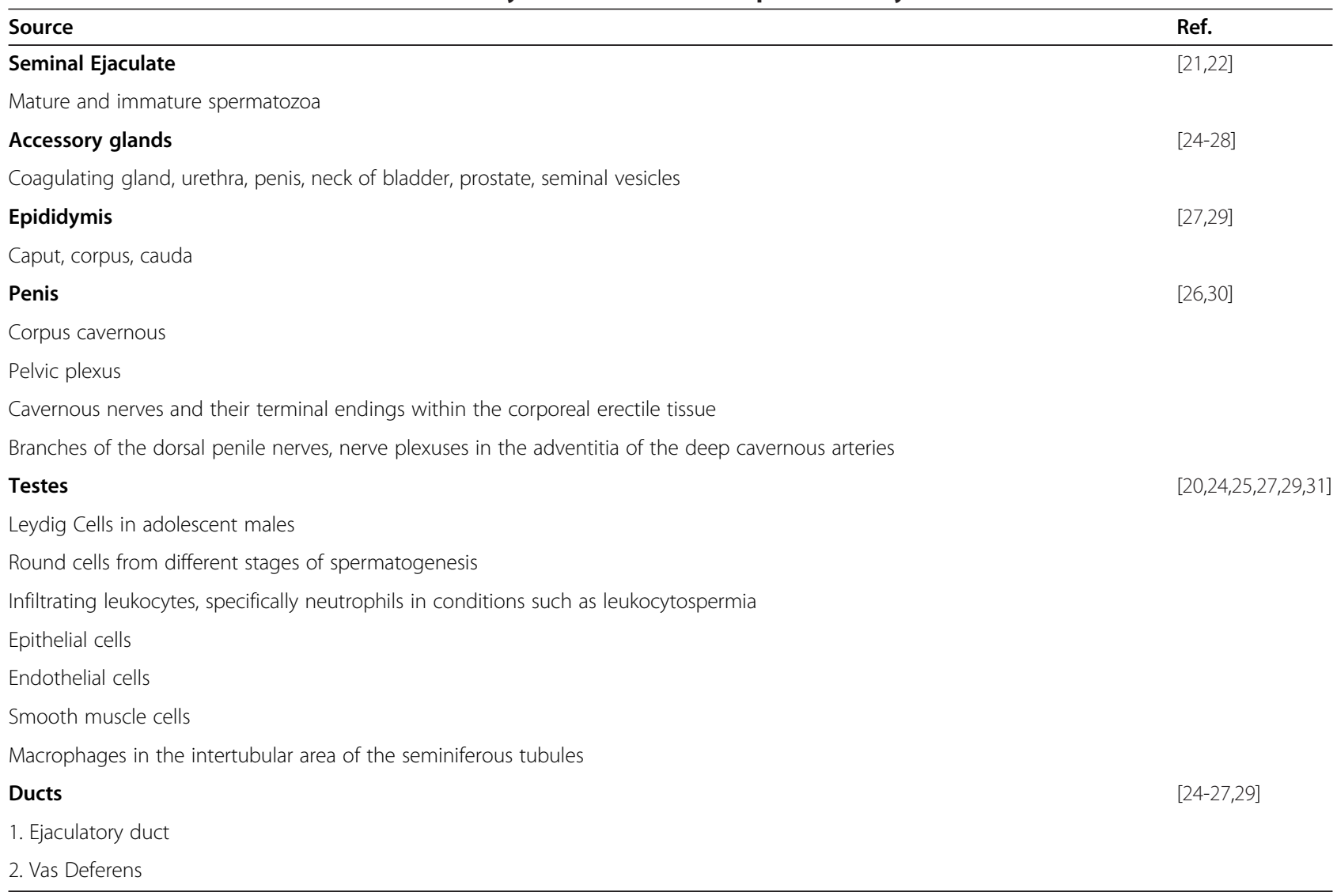




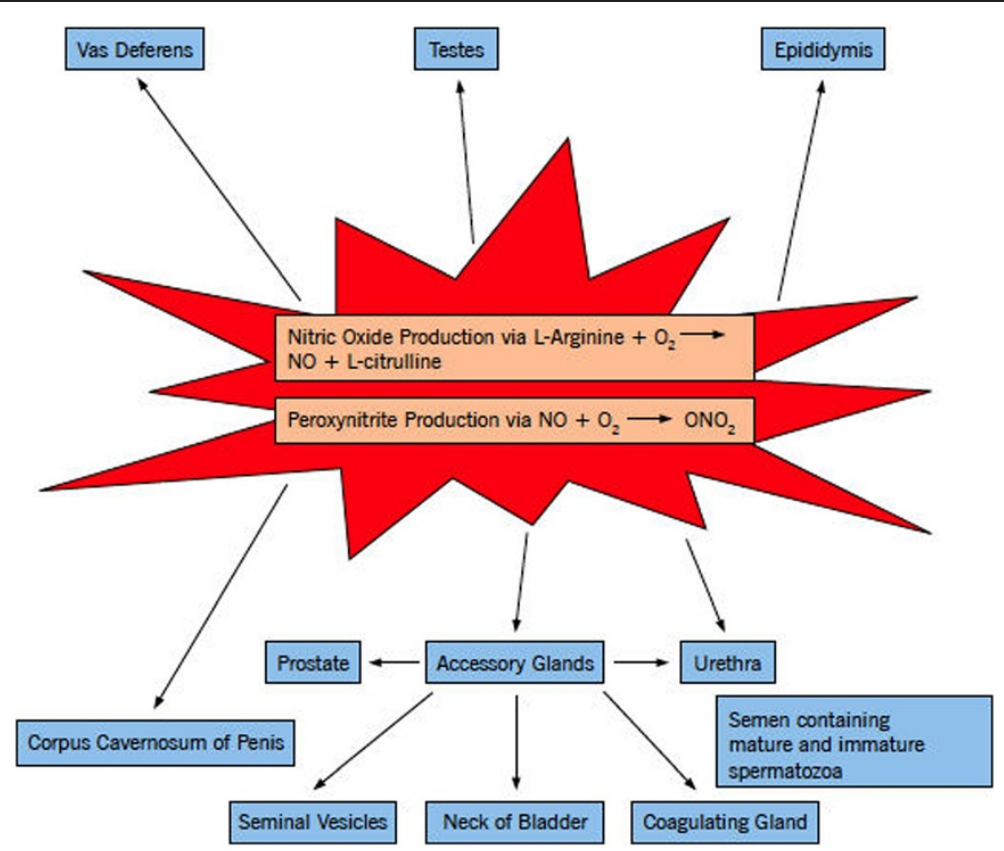

Figure 1 Sources of reactive nitrogen species. It illustrates the various locations of RNS throughout the male reproductive system. Specifically, these sources can be broken down by structure and various cell types as shown above.

Leydig cells, myofibroblasts, myoid cells, endothelial cells, and spermatozoa. The ubiquitous presence of eNOS, iNOS, and $\mathrm{nNOS}$ in the testis is indicative of the importance of NOS for spermatogenesis [9]. Aside from NOS, there are a variety of other compounds and biochemical reactions that produce NO in the body. Namely, studies have linked the generation of NO to the rate-limiting enzyme glucose-6phosphate dehydrogenase as well as the NADPHproducing pentose phosphate pathway [35].

Furthermore, glucose has been shown to indirectly produce NO by stimulating the pentose phosphate pathway as well as the conversion of L-arginine to L-citrulline. Other studies have indicated that $\mathrm{NO}$ can regulate its own activity via a feedback inhibition mechanism [24,25]. While excessive levels of NO undoubtedly damage reproductive organs, it is nevertheless one of least potent of the RNS. In particular, the reaction of NO with the superoxide anion results in the formation of a more noxious oxidant, peroxynitrite [10-12,17,18,35]. Highly toxic to sperm, peroxynitrite and its breakdown product, Peroxynitrous acid (HONOO), are capable of inducing peroxidative damage and nitrosation of tyrosine molecules that facilitate signal transduction [24,25]. These cytotoxic effects often occur via the interaction of peroxynitrite and HONOO with nucleic acids, proteins, and lipids within the spermatozoa either through direct oxidative reactions or indirect, radical-mediated mechanisms $[20,24,25]$. Usually, the formation of peroxynitrite occurs only when NO has reached toxic levels and begins to compete with superoxide dismutase for the scavenging of superoxide [9]. Other important
RNS include nitrosyl-containing compounds. Specifically, NO reacts with the thiol groups of proteins forming biologically active and stable S-nitrosyl compounds. Researchers report that production of the isoform, dinitrosyl iron cysteine, could be a possible mechanism for NO transport.

Additional pathways of nitric oxide transport can be generated via binding of $\mathrm{NO}$ to iron-sulfur clusters, formation of nitrotyrosines, and binding of NO to hemecontaining proteins of the respiratory chain [20]. Overall, by developing a better understanding of the mechanisms and effects of RNS on the body, novel therapeutic strategies can be formulated to remove these toxic compounds and thereby alleviate their harmful effects [15].

\section{Role of reactive nitrogen species}

Function of reactive nitrogen species in signal transduction

At physiologic levels, RNS are crucial for various functions within the male reproductive system. Although literature fails to elaborate on the exact levels of RNS that result in pathogenesis, studies have shown that concentrations below one micromolar play an important role in the regulation of a variety of signaling pathways $[15,16,20,31,32]$. At low levels, less than one micromolar, NO directly interacts with soluble guanyl cyclase to stimulate synthesis of cyclic guanosine monophosphate (cGMP), which in turn, activates cGMP regulated phosphodiesterase, protein kinase G, and cyclic nucleotide-gated channels. Additionally, minimal concentrations of nitric oxide, less than one micromolar, can induce mitogen activated protein (MAP) kinase signaling pathways. These pathways relay information to 
effectors, amplify signals, coordinate incoming information from other signaling pathways, and allow for a variety of response patterns [20].

\section{Role of reactive nitrogen species in the blood-testis- barrier}

While NO is clearly important for intracellular signaling pathways, it is also involved in the regulation and assembly of tight junctions within the blood-testis barrier. This barrier creates an environment in which germ cells develop via changes in the chemical composition of the luminal fluid. The blood-testis barrier also prevents passage of toxic substances into the seminiferous tubules. Specifically, NO controls the timely opening and closing of this barrier, which has been found to be essential for the processes of spermatogenesis, germ cell maturation, and development [20,31,32]. Conversely, studies show that NOS inhibitors facilitate the assembly and tightening of the Sertoli cell-tight junction barrier, preventing the passage of spermatocytes and their complete maturation from spermatogonia to haploid spermatozoa across this barrier [20]. Hence, by understanding the importance of nitric oxide in mediating tight junction dynamics, appropriate male contraceptives can be targeted to inhibit the maturation of the spermatozoa across the blood-testis barrier, thereby preventing the formation of viable sperm for pregnancy $[20,31,32]$.

\section{Function of reactive nitrogen species in the immune system and male reproductive organs}

Other physiologic roles of RNS include mediation of cytotoxic and pathological events, production of hormones, and facilitation of inflammation via prevention of platelet aggregation and adherence of neutrophils to endothelial cells [10-12,24,25,36]. RNS also contributes to normal vascular tone, which is especially important during an erection via the $\mathrm{NO} /$ guanyl cyclase/cyclic GMP signaling pathway $[4-6,17,18,26]$. Moreover, studies have found that NOS innervates the sympathetic preganglionic neurons from the spinal cord to the penis, suggesting that NO may affect penile erection at several neuronal levels $[24,25]$. In the urogenital tract, NO allows for appropriate function of the penis, urethra, and bladder, thereby suggesting its role as a physiological mediator of peripheral autonomic activity [27]. Thus, it appears that physiological amounts of NO aid in many of the baseline functions of the male reproductive system.

\section{Positive effects of nitric oxide on sperm parameters}

While physiological levels of nitric oxide are important in a variety of general body functions, they also are essential for conducting various sperm functions such as capacitation, acrosomal reaction, zona pellucida binding, as well as sperm motility, morphology, and viability [7-12]. Capacitation is an ongoing priming process that spermatozoa undergo in the female genital tract. It involves NO-mediated tyrosine phosphorylation of two sperm proteins and is marked by the influx of bicarbonate and calcium, efflux of cholesterol, and increase in $\mathrm{pH}$ and cyclic AMP [7-9,21,22]. Reports suggest that incubation of sperm with low concentrations of an NOreleasing compound, sodium nitroprusside, increases the percentage of capacitated sperm $(\mathrm{p}=0.0007)$ measured by the acrosome reaction [10-12,37]. Additionally, NO has been demonstrated to modulate lipoxygenase and cyclooxygenase activities during capacitation, implicating nitric oxide in sperm function [10-12].

In order for capacitation to occur, spermatozoa must achieve two events for proper fertilization: hyperactivated motility and the acrosome reaction [10-12,37,38]. In particular, hyperactivation of sperm is characterized by high amplitude, asymmetric flagellar movement, non-linear motility, and a propulsive force to penetrate the cells of the oocyte [7-9,39]. While many experiments demonstrate that physiological levels of $\mathrm{NO}$ increase the motility of spermatozoa and excess amounts decrease motility, some studies have failed to show significant changes in sperm motility in the presence of this compound. Specifically, two studies showed that $\mathrm{NO} / \mathrm{NO}$ variant concentrations $<1$ micromolar increased sperm motility, while six of studies showed a decrease in sperm motility at concentrations $>1$ micromolar. Additionally, one study showed that concentrations above or below one micromolar did not effect on sperm motility. Interestingly, one study in which NO concentration exceeded the physiologic parameter of one micromolar demonstrated increased motility, while one study in which $\mathrm{NO}$ concentrations fell below one micromolar decreased motility [See Table 2; Figure 2].

Closely intertwined with motility, the acrosome reaction is also influenced by the amount NO in the body. Release of proteolytic enzymes from the acrosome or cap of the spermatozoa occurs as the sperm approaches the ovum. These enzymes create an opening in the glycoprotein layer, allowing the sperm to appropriately bind and fuse with the mature egg. Studies have shown that there is an increased percentage of sperm undergoing the acrosome reaction in the presence of the NO-releasing compound, sodium nitroprusside $[10-12,37,42]$. In addition to its significance in mediating the acrosomal reaction, physiologic levels of nitric oxide are also necessary for zona pellucida binding [38]. Specifically, incubation of spermatozoa with low levels of sodium nitroprusside $\left(10^{-7}-10^{-8} \mathrm{M}\right)$ increased the binding of sperm plasma membrane to the membrane of the ovum [10-12]. Moreover, NO also contributes to normal sperm morphology, which has been shown to aid in the accurate prediction of fertility status and pregnancy outcomes during assisted reproductive techniques [48]. 
Table 2 Effects of nitric oxide levels on sperm parameters

\begin{tabular}{ll}
$\begin{array}{l}\text { Sperm } \\
\text { parameter }\end{array}$ & Concentration of sperm or NO/NOS/NO inhibitor/releas \\
\hline Motility & $50-100 \mathrm{nM}$ eNOS \\
& \\
Motility & $10^{-5} \mathrm{M} \mathrm{L-NAME}$ \\
& \\
Motility & SNP levels in infertile males with leukocytospermia \\
& $(6.58 \pm 0.5 .6 \mu \mathrm{M}) ;$ infertile males without leukocytospermia \\
& $(5.51 \pm 0.25 \mu \mathrm{M}) \mathrm{vs}$. Control (3.91 $\pm 0.16 \mu \mathrm{M})$
\end{tabular}

\begin{tabular}{|c|c|}
\hline \multirow[t]{5}{*}{ Motility } & GSNO (100 nmol/L) \\
\hline & $\mathrm{PTIO}(100 \mu \mathrm{mol} / \mathrm{L})$ \\
\hline & 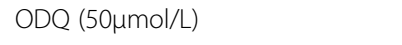 \\
\hline & 8-Br-cGMP (1 mmol/L) \\
\hline & Rp-8-Br-cGMPSs (10 $\mathrm{\mu mol} / \mathrm{L})$ \\
\hline Motility & $50 \mathrm{nM}$ and $100 \mathrm{nM}$ SNP \\
\hline Motility & $5 \mu \mathrm{M}$ GSNO \\
\hline \multirow[t]{2}{*}{ Motility } & SNP $(0.25-2.5 \mathrm{mM})$ \\
\hline & SNAP (0.012-.6 mM) \\
\hline Motility & SNAP 0-1.2 nmol/10 $10^{6}$ spermatozoa \\
\hline tility & $10^{-6}$ to $10^{-4} \mathrm{M}$ SNP \\
\hline
\end{tabular}

Morphology Good morphology $\geq$ or equal to $14 \%$ normal sperm.

Poor morphology $<14 \%$ normal

Morphology Good morphology $\geq$ or equal to $14 \%$ normal sperm

1. The maintenance of percent motility at 3 hours post-thaw was significantly improved in SNP treated samples $(p<0.05)$

1. The NO donor, GSNO, significantly increased progressive motility $(77,78$, and $78 \%$ vs 66,65 , and $62 \%$ of the control)

1. Aberrant patterns of sperm eNOS expression associated with decreased sperm motility $(r=-0.46 ; p<.05)$.

2. At low concentrations, NO improved post- thaw sperm motility.

1. In the presence of NO inhibitor, L-NAME, the percentage progressive motility, average path velocity, straight linear velocity, and curvilinear velocity were significantly reduced after $30 \mathrm{~min}$.

1. There was a significant correlation between the $\mathrm{NO}_{2}$ concentration and sperm motility $(r=0.33 ; p<0.0005)$.

2. SNP reduced the sperm motility in a dose- and timedependent manner $(\mathrm{p}<0.0001)$.

1. Higher NO concentrations result in lower total percentage of [38] sperm motility $(p<0.0007)$

1. Addition of SNP decreased mouse sperm motility, without any change in hyperactivation.

2. The NOS inhibitor, L-NAME, and the NO scavenger, methylene blue inhibited sperm motility $(p<.0 .005)$

1. A 20 minute incubation of nitric oxide with $\mathrm{NO}$ releasing compounds (GSNO, PTIO, ODQ, 8-Br-cGMP, and Rp-8-BrcGMPSs) did not alter the progressive motility of human sperm $(\mathrm{p}<0.05)$.

2. A similar effect was obtained with the NO donor sperm, NONOate, after 30 and $60 \mathrm{~min}$.

1. NO decreased sperm motility $(r=0.740 ; p<0.01)$.

1. A positive correlation was seen between the concentrations of $\mathrm{NO}$ and the percentage of immotile spermatozoa $(p<0.01)$.

1. The percentage of motile sperm, progressive motility, and concentration of motile cells were all significantly reduced with all doses of SNP $(p<0.005)$.

1. No significant difference was observed between NO production and sperm morphology (good or poor).

2. No association was found between poor semen quality and elevated levels of basal NO production.

1. A positive correlation was shown between concentrations of seminal plasma $\mathrm{NO}$ and defects in sperm morphology $(r=0.4$; $p<0.05)$.

2. Low levels of $\mathrm{NO}$ within the seminal plasma has been associated with defects in sperm morphology $(r=0.4 ; p<0.05)$.

1. Sperm viability in SNP treated sperm did not differ significantly from that of control sperm $(p>0.05)$.

1. NO has been found to reduce sperm viability $(p<0.05)$.

$\begin{array}{ll}\text { Viability } & 10^{-6}-10^{-4} \mathrm{M} \mathrm{SNP} \\ \text { Viability } & \text { SNP }(0.25-2.5 \mathrm{mM}) \\ & \text { S-nitroso-N-acetylpenicillamine (SNAP: 0.012-0.6 mM) } \\ \text { Viability } & 10^{-5} \mathrm{M} \text { L-NAME } \\ \text { Viability } & 50-100 \mathrm{nM} \mathrm{NO}\end{array}$

1. Sperm viability did not decrease in the presence of L-NAME, a nitric oxide synthase inhibitor. 
Table 2 Effects of nitric oxide levels on sperm parameters (Continued)

\begin{tabular}{|c|c|c|c|}
\hline & & $\begin{array}{l}\text { 1. At low concentrations, NO improves post-thaw sperm } \\
\text { viability. }\end{array}$ & \\
\hline Viability & $>40 \times 10^{6} \mathrm{sperm} / \mathrm{mL}$ & $\begin{array}{l}\text { 1. NO released by SNP has been shown to play a role in the } \\
\text { maintenance of sperm viability after cryopreservation. }\end{array}$ & {$[38]$} \\
\hline Viability & $10^{-4} \mathrm{M}$ SNP & 1. NO has been shown to decrease sperm viability & {$[45]$} \\
\hline \multicolumn{4}{|c|}{$\begin{array}{l}\text { L-NAME = NG-nitro-L-arginine methyl ester. } \\
\text { SNAP = S-nitroso-N-acetylpenicillamine. } \\
\text { SNP = Sodium Nitroprusside. } \\
\text { GSNO = S-Nitrosoglutathione. } \\
\text { PTIO = 2-Phenyl-4,4,5,5-tetramethylimidazoline-3-oxide-1-oxyl. } \\
\text { ODQ = 1H-[1,2,4]Oxadiazolo[4,3-a]quinoxalin-1-one. } \\
\text { 8-Br-cGMP = 8-Bromoguanosine- 3', 5'- cyclic monophosphate. } \\
\text { Rp-8-Br-cGMPSs = 8-Bromoguanosine- 3', 5'- cyclic monophosph }\end{array}$} \\
\hline
\end{tabular}

\section{Nitric oxide and its effects on genetic integrity}

Since normal levels of NO play an integral role in carrying out these aforementioned functions, it follows that excess levels of $\mathrm{NO}$ can disrupt these signaling pathways and reactions. More specifically, concentrations greater than one micromolar have been associated with a variety of detrimental effects. For instance, the mitochondrial or nuclear DNA of spermatozoa can become severely damaged via the processes of deamination, oxidation, or nitration $[20,31,49]$. DNA damage is especially injurious given the variety of unfavorable fertility outcomes that can result from such harm. Specifically, impaired conception rates, increased incidence of abortion, and genetic defects in offspring have all been linked to various forms of damage in the spermatozoan genome. Furthermore, damage may include high frequency of double and single stranded breaks, inhibition of mitochondrial respiration and DNA synthesis, chromosomal rearrangements, chromatin cross-linking, modifications of bases, and chromosome microdeletion [15,16,21,22,35].

\section{Nitric oxide and its role in apoptosis}

Other problems associated with pathological levels $\left(10^{-4} \mathrm{M}\right)$ of NO include germ cell degeneration via apoptosis [20].
Usually, apoptosis is a normal and necessary response of the male reproduction system to eliminate abnormal spermatozoa. However, high levels of NO damage the mitochondrial membrane of the sperm, inducing the release of cytochrome-c and the activation of the caspase cascade to stimulate excess apoptosis $[15,16]$.

\section{Effect of nitric oxide on steroid production}

Studies have also shown that pathologic amounts of NO, greater than one micromolar, inhibit Leydig cell steroidogenesis. While the production of male steroids is important for development of secondary sexual characteristics and semen production, it can be hindered by the disruptive effect of excess NO on cytochrome P450, which is a cholesterol side-chain cleavage enzyme [20,24,25,50]. Eventually, the lack of steroids, such as testosterone and dihydroxytestosterone, can result in male infertility and a variety of diseases such as hypogonadism, type II diabetes, and metabolic syndrome [50].

\section{Excess nitric oxide and sperm lipid peroxidation}

Toxic levels of NO, greater than one micromolar, have been implicated in lipid peroxidation of the polyunsaturated fatty

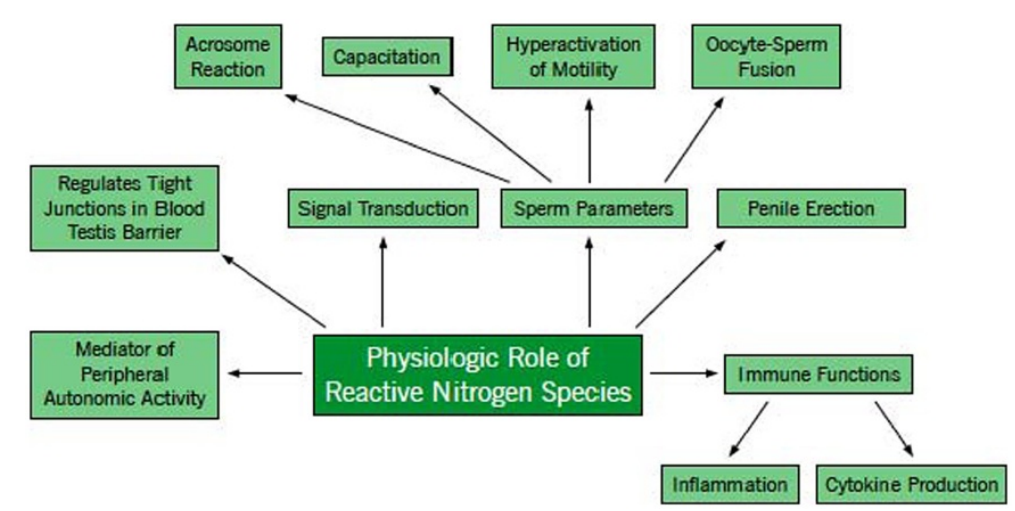

Figure 2 Physiologic role of reactive nitrogen species. It shows the essential role RNS plays in the human body. At physiologic levels, RNS helps to stimulate the immune system, maintain normal sperm parameters, and carry out general reproductive functions in the male body as shown above. 
acids (PUFA) within the sperm plasma membrane. Specifically, PUFA are very susceptible to the attack of RNS because their chemical structure contains hydrogen that can be easily abstracted. Abstraction by NO triggers a cascade of reactions, resulting in the production of a free radical that can be further oxidized to form more free radicals. This NO-mediated chain reaction is known as lipid peroxidation [15,16,51-53]. Therefore, functions of nitric oxide are highly dependent on the concentration of $\mathrm{NO}$ present in the male reproductive system.

\section{Negative effects of nitric oxide on sperm parameters}

Although studies suggest that normal levels of NO help maintain the physiologic function of the male reproductive system, higher levels have been shown to be detrimental to the various sperm parameters mentioned in the previous paragraph. Specifically, researchers report that the number of sperm bound to the zona pellucida in the presence of $10^{-4} \mathrm{M}$ sodium nitroprusside was significantly less than the control group that was treated without NO. At this same concentration of sodium nitroprusside, a considerable decrease in the viability of sperm was seen; however, a few reports failed to show any significant effect of NO on this sperm parameter [45,54] Experiments have also indicated that toxic amounts of NO result in uncharacteristic sperm appearance or morphology [See Table 2] [46]. However, similar to viability a few studies illustrated no significant effect of $\mathrm{NO}$ on sperm morphology $[39,54]$. Furthermore, higher concentrations of NO $(5.74 \pm 1.01$ microM/L) in infertile men are more likely to result in the inhibition of capacitation in comparison to the seminal plasma NO concentrations ( $3.88 \pm 0.53 \mathrm{microM} / \mathrm{L})$ of normal, fertile men. Also, in these infertile men, higher levels of $\mathrm{NO}$ were linked to a decrease in sperm metabolism [55]. Overall, it is important that the body's natural defense mechanisms properly regulate the levels of $\mathrm{NO}$ in order to prevent the detrimental effects of this compound on sperm functions [See Table 2; Figure 3].

\section{Pathological effects of nitric oxide Leukocytospermia}

Toxic levels of NO have been often coupled with leukocytospermia, characterized by the generation of oxidative stress and an excess of white blood cells in the seminal ejaculate $\left(1 \times 10^{6} \mathrm{wbc} / \mathrm{ml}\right)[45,56,57]$. Usually a contributory factor to subfertility, leukocytospermia interferes with sperm motility and causes agglutination of spermatozoa [45]. Additionally, it results in the production of cytotoxic cytokines and may be an indicator of an underlying infectious condition. Overall, leukocytospermia is often considered a major source of OS and may be the reason for higher than normal levels of nitric oxide in the body [45].

\section{Varicocele}

Given that physiological nitric oxide governs many important functions in the body, it is unsurprising that excessive or insufficient amounts of this species can contribute to a variety of diseases and disorders. In particular, high levels of NO have been associated with varicocele, which is one of the most common causes of male infertility. Varicocele is a disease characterized by swelling and widening of the veins in the pampiniform plexus along the spermatic cord, thereby inhibiting blood flow in this area $[33,58,59]$. NO, specifically formed from iNOS has been implicated in the many symptoms associated in varicocele, including testicular hypoxia, Leydig and germ cell dysfunction due to small vessel occlusion, elevation in scrotal temperature, diminished gonadotropin secretion, and testicular dysfunction. Despite these proposed disruptions in varicocele, the exact mechanistic pathway by which $\mathrm{NO}$ functions in this pathophysiology is unclear [33,34,58-61].

\section{Erectile dysfunction}

NO influences the mechanism of erectile dysfunction. Erectile Dysfunction is defined as the inability to achieve or maintain erections sufficient for satisfactory sexual intercourse [13,14,31]. Erection is mediated by the soluble

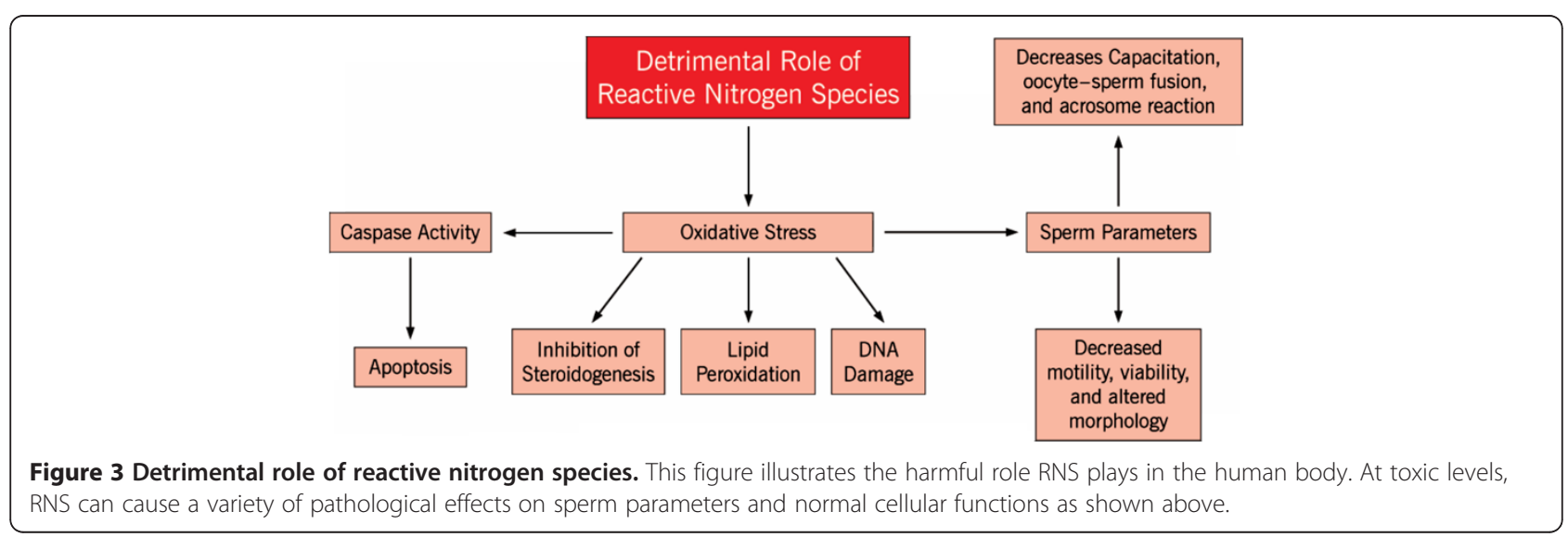


guanyl cyclase/cGMP/cGMP pathway that is activated by NO. This pathway involves the phosphorylation of a variety of proteins which leads to the relaxation of smooth muscle and filling of blood within the sinusoidal spaces of the penis. However, in erectile dysfunction, there may not be enough NO to activate this pathway because it undergoes a competing reaction with oxyhemoglobin or superoxide anion to form toxic peroxynitrite $[13,14,31]$.

\section{Diabetes mellitus}

Pathological levels of NO have been considered a causal factor in diabetes mellitus. Specifically, NO has been linked to beta cell death in the pancreas, suggesting its role in the hyperinsulinemia associated with Type I diabetes. As a result of this insulin resistance, there are increased levels of insulin in the bloodstream, which has been proposed to play a role in inhibiting spermatogenesis and male fertility $[62,63]$.

\section{Measurement of nitric oxide}

Due to the very short half-life and reactivity of NO, measurement of this compound has proved very difficult. Nevertheless, a variety of direct and indirect methods for detection of NO have been employed in the laboratory setting. Namely, these methods are of great importance due to the many clinical implications involved with excess amounts of $\mathrm{NO}$ in the body.

\section{Direct methods of nitric oxide measurement Iso-nitric oxide probe}

The Iso-NO probe is a direct NO sensor, with a probe located at the end of stainless steel sleeve. This sleeve consists of a working and counter electrode combination that is covered by a polymeric membrane, which is permeable to gas [64]. In particular, this membrane separates the semen sample from the electrode and removes interference from other dissolved gases. The removal of such interference confers a high degree of selectivity to the probe. Specifically, the reason that concentrations from one nanomolar to twenty micromolars of $\mathrm{NO}$ can be measured across the gas-permeable membrane is a result of oxidation that it undergoes as it passes through the working electrode. The oxidation of nitric oxide produces an electrical redox current that is proportional to the amount of $\mathrm{NO}$ around the polymeric membrane [39].

\section{Flow cytometry}

Flow cytometry is a method by which the intensity of fluorescence indicates the amount NO present in the sample. Specifically, NO production can be measured via changes in fluorescence which occurs after nitrosation of the NO indicator dye, diaminofluorescein-2 Diacetate (DAF-2DA). A counter stain specific to DAF-2DA is also used in order to make structures more visible [65].

\section{Indirect measurement of nitric oxide spectrophotometric measurement}

The Griess test is one of the most sensitive methods for detecting nitrite or nitrous acid. Specifically, the presence of these NO-containing compounds can be assessed by reacting them with sulfanilic acid. The resulting product is a diazonium compound, which combines with alphanaphthylamine to produce pink azo dye that is highly absorbent. In particular, the formation of this dye can be used to measure amounts of any substance that will yield nitrite in known proportions [66,67]. The manner in which the amount of nitrogenous compounds absorbed by the azo dye is measured is called spectophotometry. This method utilizes an instrument known as the spectrophotometer, which is a device for measuring light intensity as a function of wavelength as light passes through a solution. With regard to measurement of nitric oxide via spectophotometry, the NO concentration of the sperm sample is calculated according to Beer's Law. Specifically, the amount of $\mathrm{NO}$ is assessed by the graph produced by the spectrophotometer which relates absorbance to wavelength.

\section{High performance capillary electrophoresis}

High performance capillary electrophoresis separates nitrogen containing compounds based on their mass to charge ratio [68]. This method measures both nitrate and nitrite within the plasma and can be measured in a single analysis with minimal sample preparation. This method is sensitive to both basal and physiological changes in plasma nitrate and nitrite levels [68].

One of the limitations of these indirect methods is that they cannot determine the real time NO-concentration within a localized area at high spatial and temporal resolutions [66-69].

\section{Bio-imaging and electrochemical techniques}

These new techniques can allow for continuous visualization and measurement of local NO concentrations at high resolutions. The bio-imaging method utilizes an NO-specific fluorescent dye that is capable of binding with $\mathrm{NO}$ and fluoresces in a manner dependent on NO concentrations. The fluorescent images are obtained using a confocal laser scanning microscope. In the electrochemical technique, a microaxial NO-electrode and reference electrode is used to measure the amount of $\mathrm{NO}$ oxidized at the surface of the electrode apparatus. In order to successfully measure NO, a combination of a variety of analytical methods are needed to investigate the complex mechanisms and physiological effects of NO [28,67].

\section{Assessment of reactive nitrogen species by chemiluminescence}

The chemiluminescence assay utilizes an instrument known as a luminometer, in addition to a probe known as 
luminol $[12,15,16]$. Specifically, this probe is used in measurement of redox activities as well as ROS/RNS production within spermatozoa via the production of a light signal. This signal is formed from the interaction of these free radicals with the probes and it is eventually converted to an electrical signal by the luminometer [50]. Additionally, the luminol probe has the capacity to react with a variety of extracellular and intracellular ROS/RNS at a neutral $\mathrm{pH}$, and is not specific to any particular type of free radical species $[12,15,16,50]$. The results of this assay are expressed as $10^{6}$ counted photons per minute per $20 \times$ $10^{6}$ sperm or relative light units/s/106 sperm $[12,15,16]$.

\section{Conclusions}

This article describes both physiological and pathological roles of this unique subset of ROS. Studies have shown that a balance between RNS and antioxidants is undoubtedly important for a variety of functions in the male reproductive system, such as cell signaling, tight junction regulation, and production of hormones, capacitation, acrosomal reaction, sperm motility, and zona pellucida binding $[4,15,17,20,27,31]$. However, an excess of RNS can adversely affect reproductive potential by causing testicular dysfunction, decreased gonadotropin secretion, and abnormal semen parameters [24,49,51]. Because such excesses have been demonstrated in males with fertility problems and routine semen analysis has not been able to accurately predict IVF outcomes, it is imperative that novel strategies be developed in order to both assess and treat OS. One promising approach that has been proposed is an antioxidant regimen to combat excess RNS levels. Presently, however, favorable results of antioxidant treatment have only been demonstrated in patients with high levels of DNA damage in sperm. Thus, the effectiveness of antioxidant treatment should be treated with brid led optimism until further research has been conducted. Another possible option that may be therapeutically important in correcting erectile dysfunction and infertility is the utilization of NO synthesis inhibitors and donors. Hence, the determination of an ROS threshold and development of various treatments in response to increased OS will not only be beneficial from a scientific standpoint, but will also have great clinical significance as well. Since excess levels of $\mathrm{NO}$ have been associated with decreased fertilization rates, impaired embryonic development, high levels of abortion and increased morbidity in the offspring, understanding the role of RNS can potentially stem the increasing rates of male infertility and decreasing sperm quality that has been reported in recent years.

\footnotetext{
Abbreviations

cGMP: Cyclic Guanosine Monophosphate; DAF-2DA: Diaminofluorescein-2 Diacetate; eNOS: Endothelial Nitric Oxide Synthase; FAD: Flavin Adenine Dinucleotide; FMN: Flavin Monoucleotide; HONOO: Peroxynitrous acid; iNOS: Inducible Nitric Oxide Synthase; MAP: Mitogen Activated Protein;
}

nNOS: Neuronal Nitric Oxide Synthase; NADPH: Nictoninamide Adenine Dinucleotide Phosphate; NO: Nitric Oxide; NOS: Nitric Oxide Synthase; OS: Oxidative Stress; PUFA: Polyunsaturated Fatty Acids; RNS: Reactive Nitrogen Species; ROS: Reactive Oxygen Species; BH4: Tetrahydrobiopterin.

\section{Competing interests}

The authors declare that they have no competing interests.

\section{Authors' contributions}

SD participated in the original idea, carried out the literature (medline) search, compilation of the information, drafting and finalizing the paper. KK participated in compilation of the information and critical review of the paper. RKS conceived the study, participated in the study design compilation of the contents and critical review of the paper. AA provided substantial contribution ranging from study idea, design, critical review of the final paper. All authors read and approved the final manuscript.

\section{Acknowledgements}

Authors are grateful for the support from the Center for Reproductive Medicine, Cleveland Clinic.

Received: 25 September 2012 Accepted: 6 December 2012 Published: 15 December 2012

\section{References}

1. Agarwal A, Prabakaran SA: Mechanism, measurement, and prevention of oxidative stress in male reproductive physiology. Indian J Exp Biol 2005, 43:963-974.

2. Kreeger KY, Rubbo H, Radi R, Trujillo M, Telleri R, Kalyanaraman B, Barnes S, Kirk M, Freeman BA: Nitric oxide regulation of superoxide and peroxynitrite-dependent lipid peroxidation. J Biol Chem 1994, 269:26066-26075.

3. Sampson JB, Rosen H, Beckman JS: Peroxynitrite dependent tyrosine nitration catalyzed by superoxide dismutase, myeloperoxidase and horseradish peroxidase. Methods Enzvmol 1996, 269:210-218.

4. Agarwal A, Makker K, Sharma R: Clinical relevance of oxidative stress in male factor infertility: an update. Am J Reprod Immunol 2008, 59:2-11.

5. Pryor WA, Squadrito GL: The chemistry of peroxynitrite: a product from the reaction of nitric oxide with superoxide. Am J Physiol 1995, 12:L699-L722.

6. Tarpey MM, Beckman JS, Ischiropoulos H, Gore JS, Brock TA: Peroxynitrite stimulates vascular smooth muscle cell cyclic GMP synthesis. FEBS Lett 1995, 364:314-318.

7. Kothari S, Thompson A, Agarwal A, du Plessis SS: Free radicals: their beneficial and detrimental effects on sperm function. Indian J Exp Biol 2010, 48:425-435.

8. Beckman JS, Carson M, Smith CD, Koppenol WH: ALS, SOD and peroxynitrite. Nature Lond 1993, 364:584.

9. de Lamirande E, Gagnon C: Capacitation-associated production of superoxide anion by human spermatozoa. Free Radic Biol Med 1995, 18:487-495.

10. Sikka SC: Relative impact of oxidative stress on male reproductive function. Curr Med Chem 2001, 8:851-862

11. Choudhary R, Chawala VK, Soni ND, Kumar J, Vyas RK: Oxidative stress and role of antioxidants in male infertility. Pak J Physiol 2010, 6:54-59.

12. Beckman JS, Conger $\mathrm{K}$ : Direct measurement of nitric oxide in solutions with an ozone based chemiluminescent detector. Methods 1995, 7:35-39.

13. Agarwal A, Nandipati KC, Sharma RK, Zippe CD, Raina R: Role of oxidative stress in the pathophysiological mechanism of erectile dysfunction. J Androl 2006, 27:335-347.

14. Beckman JS, Beckman TW, Chen J, Marshall PA, Freeman BA: Apparent hydroxyl radical production from peroxynitrite: implications for endothelial injury by nitric oxide and superoxide. Proc. Natl. Acad. Sci. USA 1990, 87:1620-1624.

15. Makker K, Agarwal A, Sharma R: Oxidative stress \& male infertility. Indian J Med Res 2009, 129:357-367.

16. Koppenol WH, Moreno JJ, Pryor WA, Ischiropoulos H, Beckman JS: Peroxynitrite: a cloaked oxidant from superoxide and nitric oxide. Chem Res Toxicol 1992, 5:834-842.

17. Pacher P, Beckman JS, Liaudet L: Nitric oxide and peroxynitrite in health and disease. Physiol Rev 2007, 87:315-424. 
18. Jourd'heuil D, Jourd'heuil FL, Kutchukian PS, Musah RA, Wink DA, Grisham $M B$ : Reaction of superoxide and nitric oxide with peroxynitrite: implications for peroxynitrite-mediated oxidation reactions in vivo. J Biol Chem 2001, 276:28799-28805.

19. Ramya T, Misro MM, Sinha D, Nandan D, Mithal S: Altered levels of seminal nitric oxide, nitric oxide synthase, and enzymatic antioxidants and their association with sperm function in infertile subjects. Fertil Steril 2011, 95:135-140.

20. Lee NP, Cheng CY: Nitric oxide/nitric oxide synthase, spermatogenesis, and tight junction dynamics. Biol Reprod 2004, 70:267-276.

21. Baker MA, Aitken RJ: Reactive oxygen species in spermatozoa: methods for monitoring and significance for the origins of genetic disease and infertility. Reprod Biol Endocrinol 2005, 3:1-9.

22. Cornwell TL, Arnold E, Boerth NJ, Lincoln TM: Inhibition of smooth muscle cell growth by nitric oxide and activation of CAMP-dependent protein kinase by cGMP. Am J Physiol 1994, 267:C1405-C1413.

23. Zhang $H$, Zheng RL: Possible role of nitric oxide on fertile and asthenozoospermic infertile human sperm functions. Free Radic Res 1996, 25:347-354.

24. Rosselli M, Keller PJ, Dubey RK: Role of nitric oxide in the biology, physiology, and pathophysiology of reproduction. Hum Reprod Update 1998, 4:3-24.

25. Archer S: Measurement of nitric oxide in biological models. FASB J 1993, 7:349-360.

26. O'Bryan MK, Zini A, Cheng CY, Schlegel PN: Human sperm endothelial nitric oxide synthase expression: correlation with sperm motility. Fertil Steril 1998, 70:1143-1147.

27. Ehren I, Adolfsson J, Wiklund NP: Nitric oxide synthase activity in the human urogenital tract. Urol Res 1994, 22:287-290.

28. Kitamura Y, Ogawa H, Oka K: Real-time measurement of nitric oxide using a bio-imaging and an electrochemical technique. Talanta 2003, 61:717-724.

29. Uckert S, Stanarius A, Stief CG, Wolf G, Jonas U, Machtens S: Immunocytochemical distribution of nitric oxide synthase in the human seminal vesicle: a light and electron microscopical study. Urol Res 2003, 31:262-266.

30. Zini A, O'Bryan MK, Magid MS, Schlegel PN: Immunohistochemical localization of endothelial nitric oxide synthase in human testis, epididymis, and vas deferens suggests a possible role for nitric oxide in spermatogenesis, sperm maturation, and programmed cell death. Biol Reprod 1996, 55:935-941.

31. Lee NP, Cheng CY: Nitric oxide and cyclic nucleotides: their roles in junction dynamics and spermatogenesis. Adv Exp Med Biol 2008, 636:172-285.

32. Yan $\mathrm{HH}$, Cheng CY: Blood-testis barrier dynamics are regulated by an engagement/disengagement mechanism between tight and adherens junctions via peripheral adaptors. Proc Natl Acad Sci USA 2005, 102:11722-11727.

33. Santoro G, Romeo C, Impellizzeri P, Lentile R, Cutroneo G, Trimarchi F, Pedale S, Turiaco N, Gentile C: Nitric oxide synthase patterns in normal and varicocele testis in adolescents. BJU Int 2001, 88:967-973.

34. Turker Kl, Erdoğru T, Gülkesen H, Sezer C, Usta M, Ciftçioğlu A, Baykara M: The potential role of inducible nitric oxide synthase (iNOS) activity in the testicular dysfunction associated with varicocele: an experimental study. Int Urol Nephrol 2004, 36:67-72

35. Bolaños JP, Delgado-Esteban M, Herrero-Mendez A, Fernandez-Fernandez S, Almeida A: Regulation of glycolysis and pentose-phosphate pathway by nitric oxide: impact on neuronal survival. Biochim Biophys Acta 2008, 1777:789-793

36. Rosselli M, Dubey RK, Imthurn B, Macas E, Keller PJ: Effects of nitric oxide on human spermatozoa: evidence that nitric oxide decreases sperm motility and induces sperm toxicity. Hum Reprod 1995, 10:1786-1790.

37. Vanasco V, Evelson P, Boveris A, Alvarez S: In situ and real time muscle chemiluminescence determines singlet oxygen involvement in oxidative damage during endotoxemia. Chem Biol Interact 2010, 184:313-318.

38. Balercia G, Moretti S, Vignini A, Magagnini M, Mantero F, Boscaro M, Ricciardo-Lamonica G, Mazzanti L: Role of nitric oxide concentrations on human sperm motility. J Androl 2004, 25:245-249.

39. Miraglia E, Rullo ML, Bosia A, Massobrio M, Revelli A, Ghigo D: Stimulation of the nitric oxide/cyclic guanosine monophosphate signaling pathway elicits human sperm chemotaxis in vitro. Fertil Steril 2007, 87:1059-1063.
40. Lewis SE, Donnelly ET, Sterling ES, Kennedy MS, Thompson W, Chakravarthy $U$ : Nitric oxide synthase and nitrite production in human spermatozoa: evidence that endogenous nitric oxide is beneficial to sperm motility. Mol Hum Reprod 1996, 2:873-878.

41. Nobunaga T, Tokugawa $Y$, Hashimoto K, Kubota Y, Sawai K, Kimura T, Shimoya K, Takemura M, Matsuzaki N, Azuma C: Elevated nitric oxide concentration in the seminal plasma of infertile males: nitric oxide inhibits sperm motility. Am J Reprod Immunol 1996, 36:193-197.

42. Donnelly ET, Lewis SE, Thompson W, Chakravarthy U: Sperm nitric oxide and motility: the effects of nitric oxide synthase stimulation and inhibition. Mol Hum Reprod 1997, 3:755-762.

43. Hellstrom WJ, Bell M, Wang R, Sikka SC: Effect of sodium nitroprusside on sperm motility, viability, and lipid peroxidation. Fertil Steril 1994 61:1117-1122

44. Miraglia EE, De Angelis FF, Gazzano E, Hassanpour HH, Bertagna AA, Aldieri EE, Revelli AA, Ghigo DD, Alberto R: Dar: nitric oxide stimulates human sperm motility via activation of the cyclicGMP/protein kinase $\mathrm{G}$ signaling pathway. J Reprod Fertil Supp/ 2011, 141:47-54

45. Tomlinson MJ, East SJ, Barratt CL, Bolton AE, Cooke ID: Preliminary communication: possible role of reactive nitrogen intermediates in leucocyte-mediated sperm dysfunction. Am J Reprod Immunol 1992, 27:89-92.

46. Wu TP, Huang BM, Tsai HC, Lui MC, Liu MY: Effects of nitric oxide on human spermatozoa activity, fertilization and mouse embryonic development. Arch Androl 2004, 50:173-179.

47. Burnett AL, Tillman TL, Chang TS, Epstein Jl, Lowenstein CJ, Bredt DS, Snyder SH, Walsh PC: Immunohistochemical localization of nitric oxide synthase in the autonomic innervation of the penis. J Urol 1993, 150:73-76.

48. Revelli A, Costamagna C, Moffa F, Aldieri E, Ochetti S, Bosia A, Massobrio M, Lindblom B, Ghigo D: Signaling pathway of nitric oxide-induced acrosome reaction in human spermatozoa. Biol Reprod 2001, 64:1708-1712.

49. Amiri l, Sheikh N, Najafi R: Nitric oxide level in seminal plasma and its relation with sperm DNA damages. Iran Biomed J 2007, 11:259-264.

50. Aitken RJ, Baker MA, O'Bryan M: Shedding light on chemiluminescence: the application of chemiluminescence in diagnostic andrology. $J$ Androl 2004, 25:455-465.

51. Bain J: Testosterone and the aging male: to treat or not to treat? Maturitas 2010, 66:16-22

52. Dhindsa S, Prabhakar S, Sethi M, Bandyopadhyay A, Chaudhuri A, Dandona P: Frequent occurrence of hypogonadotropic hypogonadism in type 2 diabetes. J Clinical Endocrinol Metab 2004, 89:5462-5468.

53. Semenova AV, Tomilova IK, Panikratov KD, Kadykova EL, Basharin AV: The role of nitric oxide in fertility disorders in men. Urologiia 2005, 6:31-36.

54. Kruger TF, Menkveld R, Stander FS, Lombard CJ, Van der Merwe JP, van Zyl JA, Smith K: Sperm morphologic features as a prognostic factor in in vitro fertilization. Fertil Steril 1986, 46:1118-1123.

55. Burnett AL, Ricker DD, Chamness SL, Maguire MP, Crone JK, Bredt DS, Snyder SH, Chang TS: Localization of nitric oxide synthase in the reproductive organs of the male rat. Biol Reprod 1995, 52:1-7.

56. Huang I, Jones J, Khorram O: Human seminal plasma nitric oxide: correlation with sperm morphology and testosterone. Med Sci Monit 2006, 12:CR103-CR106.

57. WHO: World health organization laboratory manual for the examination of human semen and semen-cervical mucus interaction. 4th edition. Cambridge: Cambridge University Press; 1999.

58. Shiraishi $K$, Naito $K$ : Nitric oxide produced in the testis is involved in dilatation of the internal spermatic vein that compromises spermatogenesis in infertile men with varicocele. BJU Int 2007, 99:1086-1090

59. Mitropoulos D, Deliconstantinos G, Zervas A, Villiotou V, Dimopoulos C Stavrides J: Nitric oxide synthase and xanthine oxidase activities in the spermatic vein of patients with varicocele: a potential role for nitric oxide and peroxynitrite in sperm dysfunction. J Urol 1996, 156:1952-1958.

60. Ozbek E, Turkoz Y, Gokdeniz R, Davarci M, Ozugurlu F: Increased nitric oxide production in the spermatic vein of patients with varicocele. Eur Urol 2000, 37:172-175.

61. Romeo C, Lentile R, Impellizzeri P, Turiaco N, Teletta M, Antonuccio P, Basile M, Gentile C: Preliminary report on nitric oxide mediated oxidative damage in adolescent varicocele. Hum Reprod 2003, 18:26-29. 
62. Du Plessis SS, Cabler S, McAlister DA, Sabanegh E, Agarwal A: The effect of obesity on sperm disorders and male infertility. Nat Rev Urol 2010 7:153-161.

63. Agbaje IM, Rogers DA, McVicar CM, McClure N, Atkinson AB, Mallidis C Lewis SEM: Insulin dependent diabetes mellitus: implications for male reproductive function. Hum Reprod 2007, 22:1871-1877.

64. World Precision Instruments: Lab equipment for the life sciences. http://www. wpiinc.cn/pdf/iso-nop-maintenance.pdf.

65. Navarro-Antolin J, Lamas S: Nitrosative stress by cyclosporin A in the endothelium: studies with the NO-sensitive probe diaminoflurescein-2/ diacetateusing flow cytometry. Nephrol Dial Transplant 2001, 16:6-9.

66. Liebhafsky HA, Winslow EH: Spectrophotometric determination of nitrite: and of nitric oxide in furnace atmosphere. Ind Eng Chem Anal Ed 1939, 11:189-190

67. Giustarini D, Rossi R, Milzani A, Dalle-Donne I: Nitrite and nitrate measurement by griess reagent in human plasma: evaluation of interferences and standardization. Methods Enzymol 2008, 440:361-380.

68. Leone AM, Francis PL, Rhodes P, Moncada S: A rapid and simple method for the measurement of nitrite and nitrate in plasma by high performance capillary electrophoresis. Biochem Biophys Res Commun 1994, 200:951-957.

69. Gally JA, Montague PR, Reeke GM, Edelman GM: The NO hypothesis: possible effects of a short-lived, rapidly diffusible signal in the development and function of the nervous system. Proc Natl Acad Sci U S A 1990, 87:3547-3551.

doi:10.1186/1477-7827-10-109

Cite this article as: Doshi et al: Role of reactive nitrogen species in male infertility. Reproductive Biology and Endocrinology 2012 10:109.

\section{Submit your next manuscript to BioMed Central and take full advantage of:}

- Convenient online submission

- Thorough peer review

- No space constraints or color figure charges

- Immediate publication on acceptance

- Inclusion in PubMed, CAS, Scopus and Google Scholar

- Research which is freely available for redistribution 